\title{
Conditions that Predispose to the Development of HCC: The Role of Tumor Associated Fibroblasts and of microRNA
}

\author{
C. Avellini, D. Cesselli, A.P. Beltrami, M. Orsaria, \\ S. Marzinotto, F. Morassi and S. Uzzau
}

Additional information is available at the end of the chapter

http://dx.doi.org/10.5772/56951

\section{Introduction}

The high frequency of cases of hepatocellular carcinoma (HCC) led to vigorous efforts to identify the biological behavior and the pathogenetic mechanisms of this disease. The estimated 564.000 new cases worldwide and almost the same number of deaths in 2000 make it necessary to understand how to treat and control HCC [1]. This is possible acquiring a deep knowledge of the natural history of the disease, particularly of its initial steps which bring the parenchyma to the first changes and expose the cells to chronic insults, leading to a long standing disease endowed with a high risk of cancer development.

The pathogenesis of HCC is a complex phenomenon-, given the high number of variables and factors involved in liver function and of the possible pathway activation or deregulation during liver disease.

The first valuable step is based upon the death of hepatocytes: different pathological conditions able to induce hepatocyte damage and death will determine recruitment of inflammatory cells and deposition of connective tissue.

On the one hand this fact shows the relatively limited range of liver parenchyma response to various kind of damage. The structure and function of the organ are associated with necroinflammatory, fibrotic and cholestatic changes as the main phenomena which the parenchyma response to damage is able to produce.

On the other hand, this kind of elementary lesions is mostly self-maintaining: in fact, some of the main consequences of hepatocyte destruction and fibrotic parenchymal substitution are 
the changes in blood flow and in the composition and function of both extracellular matrix and microenvironment of the liver [1], which heavily influence hepatocyte life and function.

There is a strict connection between different phenomena concerning the liver in normal and pathological conditions. Chronic inflammation and fibrosis are well known as the main changes leading to neoplasia harboring in the majority of chronic liver diseases; but some insights into the mechanisms and the cellular types which can act either in organ development, or in regenerative and neoplastic processes represent one of the objective of this text. At this regard, a particular role in the relationship between microenvironment and liver cells proliferation concerns tumor associated fibroblasts (TAFs), considered critical for tumor growth and progression.

Experimental studies in solid tumors other than HCC suggest a possible origin of these cells from mesenchymal stem cells derived from the bone marrow, but in vitro human models lack. These cells exert important functions in cirrhotic and neoplastic liver parenchyma: recently it has been demonstrated the presence in human neoplastic livers of a population of multipotent adult stem cells with properties of tumor associated fibroblasts, while a population of MASCs derived TAFs is already present in cirrhotic, not yet neoplastic parenchyma. Furthermore, mesenchymal stem cells isolated from non-neoplastic and non-cirrhotic livers can acquire a TAF phenotype when grown in a medium conditioned by tumor cell lines [2]

After a brief evaluation of the main factors playing important roles in the pathogenesis and progression of hepatocellular carcinoma (pathways deregulation with respect to morphological changes, molecular factors and pathways cross-talk influencing interplay between different cellular elements, role of extracellular matrix and angiogenesis), the relationship between liver embryogenesis, regeneration and neoplastic growths is considered, inparticular as far as the interplay between parenchymal cells and stromal component in non-neoplastic and neoplastic liver is concerned. Further deeper look is addressed to more recent progresses, which can really offer new important insights in the pathogenesis and treatment of hepatocellular carcinoma: microRNA deregulation and tumor associated fibroblasts. The former show a great potential in targeting a lot of genes involved in the neoplastic process: different tumors in different organs show strict relationships with the effects of groups of miRNA similarly associated for some organs, giving rise to signatures characteristic of neoplastic development, able to predict prognosis and to represent targets for new therapeutic chances. Similar results come from the action of miRNA in hepatocellular carcinoma, where distinct tumor subtypes are associated with different miRNA signature.

Tumor associated fibroblasts represent a population of stromal cells able to influence in crucial ways the parenchymal microenvironment changes which accompany all steps of HCC development, from the origin to progression and diffusion.

\subsection{Growth factors and molecular pathways}

With the ongoing of the pathological process the proliferation of liver cells accelerates and monoclonal populations occur: this is the hallmark of all preneoplastic lesions and takes places 
also in HCC. From this point of view the foci of phenotypically altered hepatocytes represent the first discrete step, morphologically identifiable, in the way leading to cancer.

The foci of altered hepatocytes contain differentiated hepatocytes that have acquired molecular aberrations and changed their metabolic properties [3]: this may allow the progress to malignant phenotype. These cells and their morphological and biochemical phenotype are unstable and, given the absence of uniformity in foci, the clonal origin is partly suggested: at least 8 cytomorphological and cytochemical atypical foci types exist [3]. These premalignant changes represent perhaps one of the first effects of viral or environmental damage.

The main etiological agents of HCC are well known: HBV,HCV and Aflatoxin B1 are considered causative agents of about $80 \%$ of cases [1]. During last years a lot of genomic aberrations have been identified and the mechanisms of interaction at molecular level between liver cells and etiologic agents in producing gene changes responsible of the development of neoplasia have been studied and often understood [1].

After the production of foci of altered hepatocytes, the subsequent step, morphologically defined, is represented by the formation of dysplastic foci and dysplastic nodules: in these lesions, probably, molecular changes can take further development with a selection of genetic and epigenetic changes leading to a proliferative advantage for altered hepatocytes and to the involvement of several regulatory pathways in simultaneous disorders favoring the passage from dysplastic to neoplastic lesion.

The simple increase in cell proliferation and DNA synthesis associated with increasing enzymatic and metabolic changes is not enough to give rise to carcinogenesis.

The production of growth factors and hormones (e.g. Insulin, IGF2, TGF alfa, TGF beta, HGF, Transferrin, VEGF), of cytokines and chemokines and the activation or deregulation of different pathways give an essential contribution to the development of neoplasia, but the whole mechanism of the transformation from dysplastic to fully neoplastic lesion is not yet completely understood.

A crucial element to consider in this context comes from the interaction between the different cell types present in liver parenchyma.

For example, hepatocyte proliferation is regulated by cytokines (especially IL-6) produced by Kupffer cells as well as by endothelial cells and hepatic stellate cells. On the other hand, hepatocytes modulate sinusoidal endothelium phenotype and its production of prostaglandins, endothelin, IL-1 e IL-6 and Hepatocyte Growth Factor [4]. Moreover, the complexity of cross talk between cellular types is relevant in the relationship between hepatocytes and hepatic stellate cells: the production of peptides stimulating hepatic stellate cells proliferation leads to the increased production of cytokines by hepatic stellate cells. These latter are able either to inhibit or to stimulate hepatocyte proliferation [5]. For example, TGF-beta produced by hepatic stellate cells can act as an inhibitor of liver cells replication; moreover, IL-6 response is downregulated by SOCS-3 (suppressor of cytokine signaling 3) with blocking of JAK mediated STAT-3 activation $[4,5]$. 
Regarding the interaction between hepatocytes and Kupffer cells, growth factors and cytokines activate critical transcription factors, such as AP-1, NFkB, STAT-3, inducing Kupffer cells activation and production of IL-6 thus increasing liver cell DNA synthesis [5].

\subsection{Extracellular matrix}

The site where the described interactions take place is not a silent structure away from any biochemical activity: the relationship between hepatocytes and extracellular matrix is particularly active and crucial [3].

A lot of adhesion molecules and receptor mediating cell-matrix binding are present.

Extracellular matrix acts as a reservoir and presenter of cell growth factors and cytokines; it undergoes a rapid turnover and significant modifications, often induced by liver parenchymal and non-parenchymal cells, through the production of matrix-degrading enzymes, such as metalloproteinases, and their inhibitors which control the extracellular matrix degradation. Soluble mediators able to influence hepatic stellate cells and Kupffer cells induce the production of proteinases which may initiate the process of matrix degradation. Kupffer cells adhesion on endothelial cells via CD4 and ICAM-1 produce hepatic stellate cells stimulating factors: again, hepatic stellate cells, modulated by hepatocytes, endothelial cells, Kupffer cells, platelets and inflammatory cells play a role in fibrogenesis and liver morphogenesis.

When extracellular matrix at sinusoidal subendothelial level undergoes some kind of disruption, hepatocytes can lose their differentiated function and morphofunctional changes take place: altered porosity in sinusoidal barrier and impaired movement of solutes and macromolecules into and out of Disse spaces favor fibrogenetic progression, with further functional impairment. In particular, extracellular matrix turnover and degradation are under the control of different factors, either at extracellular (metalloproteinases) or intracellular level (lysosomalcathepsins) [3].

Collagenases acting on fibrillar collagen, stromelysins (degrading collagen IV, gelatin, laminin, fibronectin), gelatinase, neutrophilic collagenase ( acting on collagen I and III) and so on parallel the activity of molecules expressed by hepatocytes and hepatic stellate cells, functioning as inhibitors of metalloproteinases (tissue inhibitors of metalloproteinases 1 and 2 and alfa 2 macroglobulin), with a regulation of relative gene expression by same cytokines and growth factors as metalloproteinases [3].

Interstitial collagenase activity is increased in liver fibrosis, mainly during the early development of extensive lesions, but diminishes with advanced cirrhosis.

\subsection{Angiogenesis}

Fibrogenetic progression with Disse spaces fibrosis and sinusoidal capillarization induces great impairment particularly in hepatocellular secretion of proteins (albumin, clotting factors, lipoproteins), associated with dramatic changes in fluid dynamics [3].

Genetic changes and local hypoxia may lead to secretion of soluble angiogenic factors, with a complex interplay between cells, basal membranes and pro-or antiangiogenic factors. 
The proangiogenetic factors exert their influence in particular by activating endothelial cells, which, when activated, loose interendothelial cells contacts and breakdown the surrounding basement membrane and extracellular matrix: the associated phenomena of proliferation and migration of endothelial cells are widened by further secretion of angiogenic factors,formerlysequestered in perivascular extracellular matrix. The secretion of protease induces the release of free vascular endothelial growth factor which stimulates endothelial cells [6, 7].

Endothelial cells proliferation and migration is followed by the tendency to assemble in tubular structures, with subsequent basement membrane material production and pericytes (supportive vascular smooth muscle cells) recruitment through the action of PDGF beta: these newly formed vascular channels nevertheless show irregular and variable diameter, with abnormal branching pattern, incomplete structure of basement membrane and only partialpericytes cover, which represent the main characteristics of new vascular channels associated with the development of neoplastic growth at the beginning. The process go on when phenomena ofhypoxia in the central area of the tumoror some viral component if present ( for example HBX protein ) stimulate the production of Hipoxia Inducible Factor-1alfa, followed by the action of VEGF glycoproteins (A,B,C,D and placental growth factor) on corresponding receptors (flt1 and flk1) on endothelial cells. It has been demonstrated that Trans arterial chemoembolization (TACE) for hepatocellular carcinoma treatment is followed by parallel increase of VEGF expression related to microvascular density and hypoxia [6,7], which confirms the relationship between ischemia and reactive neoangiogenesis.

Angiogenic stimuli produce different effects in normal, cirrhotic and neoplastic liver parenchyma:within normal and regenerating tissue new functional sinusoids appear, while in chronic liver disease capillarized vascular structures take place [7].

The high number of complex interplays, here rapidly summarized, underlines the crucial connection between parenchymal cells, non-parenchymal cells and extracellular matrix, including vascular component. Again this phenomenon-show significantsimilarities between different physiological and pathological events involving the liver.

\section{Liver development, regeneration, neoplasia: The role of stem cells}

It has been recognized the existence, in the liver, of a strict connection between development, regeneration, and carcinogenesis [8,9]. As a consequence, researchers are trying to dissect the molecular mechanisms regulating liver homeostasis, the comprehension of which could open the way to new targeted therapies for liver regeneration, liver cirrhosis and primary liver cancers $[8,9]$.

Liver development involves complex mechanisms. Multipotent tissue specific progenitor cells, derived from blastocyst inner cell mass stem cells, give rise to the different organs [8]. Specifically, fibroblast growth factor (FGF) from the cardiac mesoderm, through a coordination of signaling with bone morphogenic proteins from the septum transversum mesenchyme, 
influences the hepatic induction [10-14]. In fact, committed foregut endoderm elements, through WNT signaling, promote liver bud emergence [15-17]: hepatoblasts invade the septum transversum mesenchyme to give rise to liver bud and proliferate under the influence of mesenchymal cells derived cytokines and growth factors, such as FGF, HGF and TGF beta [18-21] and of neighboring endothelial cells signaling.

The hepatoblasts show bipotential characteristic and specific pathways induce their differentiation into hepatocytes or biliary epithelium, respectively. Some studies suggest the ductal plate as the site of fetal liver progenitor cells, with 4 hypothetical anatomic compartments [22]: hepatocytes which meet the canal of Hering, pankeratin positive cells in the canal of Hering, intraductalcholagiocytes, peribiliary “ null” cells.

After injury, liver regeneration processes are strictly related to the extent of the loss of liver parenchyma [8] Specifically, after experimental partial hepatectomy, mass restoration is mainly due to mitotic division of mature liver cells [23], while when this regenerative capacity is overwhelmed by massive parenchymal loss, for example in chronic liver disease, mass regeneration implies the activation of a liver progenitor cell compartment [22]. The molecular pathways activated in this latter case suggest a recapitulation of the fetal development [8]. In fact progenitor cells phenotypes and antigenic profiling are similar to fetal liver: these cells are located at the canal of Hering; the presence of label retaining cells within the iuxta portal, proximal biliary tree (slow cycling cells which retain Bromodeoxyuridin (BRDU) label for 8 weeks after experimental injury related cell division) confirm this site as a possible stem cell niche [22]. Similar observations concern peribiliary hepatocytes, mostly identified as BRDU positive very early in post-injury period, at variance with mid-acinar and central acinar hepatocytes, involved in rapid turnover for regenerative response to the loss of parenchyma. On the other hand, intraductalcholangiocytes and peribiliary "null" cells are less likely to take a role in the niche population, due also to the difficult evaluation of their relationship with neighbor liver parenchyma [22].

Liver regeneration takes places within some days or few weeks from the insult. After experimental partial hepatectomy, inflammatory cells produce TNF-alfa which damages surviving cells, but induces DNA synthesis and adult liver cells proliferation; this mechanism needs the presence of a specific receptor on hepatocytes (Tumor necrosis factor Receptor 1). TNFR1-null mice can nevertheless grow to adulthood, demonstrating the possible development also in the absence of this interaction TNF alfa-TNFR1. This is simply one out of several mechanisms (such as the action of drugs or hormones) which increase hepatocyte proliferation, while interaction with TNF is not a unique way for liver development [24]: although TNF production and Glutation (GSH) synthesis represent rapid reaction to experimental hepatectomy, TNF simply activates different factors (such as NFKB and SEK-1, a stress related kinase) that permit hepatocytes to survive to TNF exposure. "Surviving hepatocytes are then surveyed for damage and repaired or deleted" [24]: this is a defensive, initial mechanism that, together with antiapoptotic and antioxidant factors, makepossible subsequent parenchymal regeneration. All these observations show a complex picture of stress regulated intracellular signals that allow adult hepatocytes to proliferate, to survive without proliferation or to dye. 
Chronic inflammatory response, growth factors and DNA damaging agents (including ROS) also play a role $[25,26]$, favoring the involvement of progenitor cells in regenerative / proliferative activity and therefore probably also favoring tumor development.

Type and duration of exposure to ROS, increasing after cellular stress, play a pivotal role in choosing the direction of cells destiny. An acute and transient increase in ROS is necessary for proto-oncogenes and growth factors to induce cellular proliferation [24]. Interestingly, similarly toregenerative response, with H-RAS mediated proliferative activity, malignant transformation also requires K-RAS proto-oncogene activation, inducing ERK 1-2 activation [24]. Surviving hepatocytes surveillance could therefore be overwhelmed by stress induced proliferative stimuli associated to malignant transformation.

On the one hand, normal livers, which undergone experimental hepatectomy, show a regenerative, mitotic response starting from adult hepatocytes. On the other hand, in the presence of a chronic parenchymal damage, the response isbased upon progenitor cells, with molecular signaling patterns that suggest a recapitulation of fetal development [8].

A part from experimental parenchymal damage, chronic ethanol consumption generally increases the death rate of mature hepatocytes. This stimulates a compensatory regenerative response to preserve normal liver mass and function. Nevertheless, most of the mature hepatocytes that survive in alcohol damaged livers are replicatively senescent and hence incapable of proliferating to replace their dead neighbors, given the chronic alcohol exposure which inhibits the induction of DNA synthesis. Hence, regeneration of alcohol damaged livers involves the expansion and differentiation of facultative liver progenitor cells [24]. This regenerative mechanism requires lengthening the time needed for liver mass reconstitution, due to the time necessary for differentiation of progenitor cells.

On the whole, the reparative processes take advantage of two principal strategies of defense, depending on the extent of the damage [8]. While mild injury is mainly repaired through compensatory hyperplasia of hepatocytes, severe damage implies the activation of a liver progenitor cell compartment [27]. In both cases, other non-parenchymal cells (stellate cells, vascular and biliary cells) proliferate as soon as hepatocytes starts to [27] and cooperate to restore morphofunctional competent liver tissue. In rodent experimental models with chronic liver injury.

HGF and EGF promote the upregulation of proliferation rate and expansion of oval cells, while TGF-beta exerts the opposite effect on oval cells [28-31], with an upregulation of the WNT-Beta Catenin pathway in acute and chronic liver injury experimental models [8]. These data again suggest symmetry between fetal development and regenerative mechanisms. Molecular mechanisms involved in fetal development, when identified, can allow to target pathways connected with parenchymal damage: tyrosine kinase inhibitors, for instance, are able to inhibit progenitor cell response, liver fibrosis and liver cancer development in a mouse model of chronic liver injury [32]; C-kit inhibition by imatinibmesylate attenuates progenitor cell expansion and inhibits liver tumor formation in mice [32]. 
Therefore, the presence of pathways playing a role either in fetal development or in regenerative but also in neoplastic phenomena opens important perspectives: three distinct cell lineages can be found in the liver,susceptible of neoplastic transformation: mature hepatocytes, small proliferating hepatocytes and stem cells [33, 34].

Specifically, hepatocellular carcinoma might result from dedifferentiation of mature hepatocytes, from activation of oval cells, from arrested differentiation of tissue based stem cells, the latter being an expression of blocked ontogeny, linked to hepatocarcinogenesis. Evidence of the fact that hepatocarcinogenesis partly recapitulates fetal development comes from some observations: both progenitor and fetal cells are self-renewing, with heterogeneous progeny and limitless division; bipotent cells, with hepatocytic and cholangiocytic potential are present in fetal livers and have been isolated in a number of HCC cell lines; finally, the strict relationship between tumor cells and fetal program is testified by the fact that HCC cell lines (e.g. Huh1, Huh 7, Hep 3b cell lines) share with fetal liver progenitors several oncofetal markers and that HCC characterized by a gene expression profile similar to fetal hepatoblasts have a poorer prognosis than HCC with an adult-type genomic profile [35-39].

According to the possible origin from progenitor cells or from dedifferentiated adult cells, HCC could assume different phenotypes and be linked to the activation of different pathways, associated to variable parenchymal morphological changes. For instance, significantly fewer HCCs expressing CK7 or CK19 show nuclear beta Catenin expression. The beta catenin pathway could preferentially involve "mature" hepatocytes versus less "mature" (progenitor) phenotype cells, with CK7 and CK19 expression, associated withless advanced fibrosis in nontumoral parenchyma [40].

CK19 expression in HCC can change with different expression of beta catenin: decreased fibrosis degree in the non-tumoral parenchyma parallels reduced nuclear beta catenin expression [25].

Regarding oval cells, many of the compounds that induce their proliferation are DNAdamaging agents or carcinogens, therefore oval cells can be considered as potential precancerous cells [41]. Oval cells activation with "ductular reaction" represent the expansion of a transit amplifyingcell compartment of small biliary bipotent cells [25].

In cirrhosis, hepatocytes are characterized by senescence determined by telomere shortening, while mesenchymal cells (e.g. endothelial cells and stellate cells) seem not to be affected by replicative senescence [25]. In the hepatocytes this latter can be the result of an enhanced proliferation rate that can persist for 20-30 years of chronic liver disease. It is possible to hypothesize that the parallel proliferative activity of progenitor cells and mesenchymal cells allows an influence of the latter elements on the progenitor cells carcinogenic evolution: it is known that sinusoidal lining cells like hepatic stellate cells proliferate in close anatomical relationship with progenitor cells and are able to produce growth factors for which progenitor cells have the receptors, suggesting an interaction between these cell compartments [26].

"A characteristic of stem cells is to survive to toxic and hypoxic stimuli due to their low cell cycling ". 
"One possibility is that HLSC may represent a mesenchymal population modified by the influence of the local environment, reflecting the importance of the niche in establishing the phenotype of MSC" [42].

The "tumor stroma" can be considered as "normal wound healing gone awry [43], able to interact through paracrine and juxtacrine pathways with the tumor stem cells that are influenced by the microenvironment in which they are endowed, with a direct role of microenvironment cells in determining the malignant phenotype [44-52]. In summary, fetal development, regeneration and carcinogenetic processes show some point of similarity in molecular pathways and mechanisms, partly recapitulatedin chronic parenchymal liver damage evolving to advanced stages.

Starting from the concept of chronic liver disease development, with progressive liver cell destruction, reactive fibrosis and general parenchymal organization disarray, it is possible a better comprehension of the whole changes.

These phenomena are all present since the beginning of liver damage, when liver cells are involved by viral or toxic damage and fibrous tissue deposition replace the destroyed cells. This is the beginning of fibrosis development, firstly at sub-sinusoidal level, then within the damaged areas, often associated, in more advanced stages, with a ductular reaction.

Importantly, evolution of liver fibrosis and activation of hepatic stellate cells share some pathways [4]. In fact, the adhesion molecules and receptors mediating cell-extracellular matrix binding and growth factors and cytokines present in ECM influence matrix turnover and modifications and, together with liver cells, endothelium, Kupffer cells and inflammatory cells, modulate hepatic stellate cells. These latter express Integrins, mediating cell adhesion to fibronectin and collagens and modulating metalloproteinases: integrins activate intracellular signaling pathways in response to ECM protein they recognize, influencing hepatocytes differentiation.

As a consequence, any kind of liver parenchymal damage, though mild, induces a regenerative liver cell response, starting from periportal liver cells [25].

Liver cells, ductular cells, stromal cells and perisinusoidal cells all undergo regenerative phenomena: it is a complex sequence associated with progressive collagen deposition with a healing role, involving subsinusoidalDisse spaces, periportal and perilaminarinterstitium, with ECM and parenchymal cells activation and proliferation and with ECM synthesis by stellate cells, fibroblasts and, in some cases, parenchymal cells. In fact, in this context, epithelialmesenchymal transition and mesenchymal-epithelial transition play a role, with different tissue component phenotypic changes, significantly involving parenchymal structure and function [53].

If chronic inflammation with long standing tissue damage represents a risk condition for neoplastic disease, cirrhosis is the typical predisposing setting for hepatocellular carcinoma. In fact, cirrhosis is characterized by a microenvironment possibly favoring HCC onset: most cases of hepatocellular carcinoma are linked to the presence of cirrhosis [54, 55]. 


\section{Molecular changes in the development of HCC: The novel role of miRNA}

During the last years it has been shown a significant correspondence between clinical and humoral parameters (tumor size, differentiation grading, HBV or HCV infection, serum alfafeto protein (AFP)) and different molecular pathways activated in HCC cases: beta-catenin and Axin-1 mutated cases, with early relapse, behave in a different way compared to cases with c-Myc and AKT activation associated with Interferon target genes inhibition, but also compared to hepatocellular carcinoma with overexpression of p53 and p21, the latter showing better differentiation and lower size [56]. Subclasses S1,S2,S3 defined on the basis of above mentioned clinical, humoraland morphological parametersare oftenassociated with different molecular profiles: S1 show Beta catenin and Axin mutation; S2 activation of c-Myc, AFP overexpression, AKT activation, IFN target genes inhibition; S3 cases are associated with better histological differentiation, overexpression of p53, p21, gene related with glycolipidic and alcohol metabolism, oxygen radical scavenging and coagulation [56]. The effort is the identification of a classification system on the basis or with the contribution of the molecular changes of the tumors.

At this regard, miRNAs can play a crucial role. In fact, each of these small endogenous non-coding RNAs is characterized by the ability to transcriptionally or post-transcriptionally regulate many different target genes, thus being responsible of complex molecular changes [57].

In fact, these sequences favor mRNA degradation through sequence-specific interaction with the $3^{\prime}$ untranslated region (3'-UTR) of targeted mRNAs; when the sequence is totally complementary, miRNA pairing will induce mRNA degradation and are involved in development, apoptosis, proliferation and differentiation processes [58]. Alternatively, sequences partially complementary will more probably induce a stop in translation, without mRNA degradation. Therefore, a single miRNA is able to regulate the genic expression of hundreds of target genes.Its altered expression could cause a "post transcriptional collapse", i.e. the contemporary deregulation of multiple tumor suppressor or oncogenes whose sequences are complementary to the considered miRNA [59], thus deregulating many molecular pathways which could favor a malignant cellular phenotype [57]. In fact, the global effect of inactivation of a miRNA molecule will be the over-expression of its target genes, while its activation will induce the down-regulation of a lot of target genes. If deregulated miRNA target genes are involved in the regulation of important biological processes, such as apoptosis, cell cycle, tumoral cells invasiveness or angiogenesis, then the risk of uncontrolled growth and tumor development will increase [58].

Accordingly, in HCC has been described a deregulation of miRNA expression [57]. The fact that HCC-related changes in miRNA expression are absent in non-neoplastic parenchyma and the association of these changes with other neoplasms confirm the hypothesis of miRNA involvement in HCC pathogenesis.

Interestingly, more than $50 \%$ of miRNA genes are located in fragile chromosomal site or in cancer-associated genomic regions [60]. 
Many experimental evidences support the relationship between fragile sites and DNA instability in cancerous cells. In fact, fragile sites are preferential sites for chromatids exchanges, translocations, deletions, gene amplification or integrations of associated viral sequences, such as HPV [60].

Besides the association with fragile sites, microRNA genes may be involved in tumorigenesis process through other mechanisms, such as point mutation, deletion, amplification, translocation or epigenetic modifications [61]. Most known microRNA have been identified within genomic cancer-associated regions: minimal regions of loss of heterozigosity, where often oncosuppressor genes are present or minimal region of amplification, where often oncogenes have been identified [62]. This strongly suggest that miRNAgenes may behave either as oncogenes or as tumor suppressor genes and, in particular, the same miRNA may behave in different way on the basis of the kind of alteration, cellular type or transcriptional/posttranscriptional regulation of target genes.

miRNA deregulation can also be the consequence of epigenetic changes, In fact, an extensive genomic analysis of miRNA-coding gene sequences showed that about $50 \%$ of these loci are CpG island associated [62] makingpossible that an alteration in DNA methylation/acethylation could be responsible for miRNA deregulation in tumors [63].

Regarding the changes in miRNA expression in different tumors, the Volinia study [64], performed a microarray analysis of 20 different miRNAs in 540 specimen obtained from different tumor types (lung, breast, stomach, prostate, colon, pancreas) identifying a miRNA signature common to some different solid tumors). The value of this study, find a confirmation in the similar grouping, between different tumors, of the miRNA expression signature: this suggest a common mechanism of involvement of miRNA in human carcinogenesis. Prostate, colon, stomach, pancreas show mostly similar signature among them, whereas lung and breast are represented by a fairly different signature. Furthermore, within the signature, some miRNA have been found whose association with other human tumors was already known: among them there are miR-17-5p, miR-20a, miR-21, miR-92, miR-106a, e miR-155, and molecular targets of these miRNA are significantly rich in tumor suppressor genes and oncogenes. These data suggest the crucial involvement of miRNA in the pathogenesis of solid tumors and confirm the function of miRNA as dominant and recessive tumoral genes. Furthermore, it has been shown the role of miRNA not only in the early phases of primitive tumors development, but also during the progression and the metastatic diffusion of the neoplastic disease. In fact, many experimental evidences show miRNA involvement in the regulation of biological process leading to the acquisition of metastatic potential, such as adhesion, invasion, migration, epithelial-to-mesenchymal transition and angiogenesis $[65,66]$.

Among miRNAs with an aberrant expression in both HCC and other solid tumors, we must list: miR221/222 (up-regulated in HCC, colon, pancreas and gastric cancer); miR-21 (upregulated in HCC, ovarian, lung, breast cancer and glioblastoma); miR-199a, 200b and 214 (down-regulated in HCC and ovarian cancer) and miR-199b (down-regulated in HCC, ovarian and lung cancer). Importantly, genes frequently de-regulated in HCC, such as p27/CDKN1B 
and p57/CDKN1C or PTEN, have been identified as targets of miR-221/222 and miR-21 targets (see below).

Considering HCC-specific miRNAs, miR-122 is one of the best characterized molecules. miR-122 is, in fact, a liver specific miRNA, representing $70 \%$ of all liver expressed miRNAs, and it is down-regulated in most human and murine HCC. Its deregulation induces important changes in the phenotype of adult hepatocytes and in several liver functions, such as lipid metabolism [67] and cholesterol synthesis [68]. These data allow hypothesizing a correlation between the lowered miR-122 expression in HCC and the loss of hepatic differentiation in neoplastic cells. Furthermore, Joplinget alhave demonstrated that miR-122 is able to bind to $5^{\prime}$ UTR RNA region of HCV [69]: this region is preserved in all six viral genotypes, so suggesting that miR-122 is an essential element for virus replication in hepatocytes. It has been shown that functional inactivation of miR-122 induces the $80 \%$ reduction of viral replication, suggesting that miR-122 inactivation in hepatocellular carcinoma could increase neoplastic cells resistance to HCV replication.

Concerning the miRNA involvement in different steps of hepatocellular carcinoma progression, it has been demonstrated a correlation between miR-222, miR-106, miR-92, miR-17-5p, miR-20, miR-18 and hepatocellular carcinoma differentiation grading, suggesting the involvement of a restricted number of miRNA in neoplastic progression [70]. According to this data a 20 miRNAs signature has been reported [71], capable to distinguish primitive hepatocellular carcinoma metastasizing through venous channels from solitary, non-metastatic tumors. Furthermore, a predictive analysis revealed that most of 20 miRNA of HCC signature are associated with patients' survival. These signatures could then represent a simple method for a diagnostic/prognostic profiling, capable of identifying HCC patients at high risk of developing a metastatic disease or a liver relapse.

Other authors evaluate the predictive accuracy in distinguishing neoplastic and non-neoplastic parenchyma equal to $97,8 \%$ on the basis of 8 miRNAs, while others again identify 18 overexpressed miRNA in hepatocellular carcinoma and only 6 overexpressedin non-neoplastic tissue [72] (and with aberrant miR-21 expression associated with metastasis risk and connected with PTEN targeting; miR-224 could act on genes apoptosis regulating (API-5) [73, 74].

Another miRNA involved in tumor progression and diffusion is miR-34a, a transcriptional target of p53, deleted in many human neoplastic lesions. Besides molecular targets involved in cell cycle progression, miR-34a regulates the expression of the oncoprotein c-Met, a tyrosin kinase receptor activated by the hepatocyte growth factor binding, able to induce the phosphorilation of molecules responsible for signal transduction, such as ERK1/2, thus exerting the function of key factors in tumor invasion and migration regulation.

A recent study [66] reported a significant reduction of miR-34a expression in most carcinomatous liver tissue with respect to non-neoplastic adjacent liver tissue, with an inverse correlation between miR-34a-and c-Met-expression levels. Furthermore, low levels of miR-34a positively relate with the development of metastatic disease and neoplastic vascular invasion. Accordingly, in vitro studies showed a lower migration and metastatic potential of neoplastic cells after miR-34a c-Met dependent suppression. 
It is worth noting that another mi-RNA under-expressed in most HCC [75], miR-199a-3p, is capable of regulating c-Met oncogene expression [76]:these data suggest that various deregulated miRNA in a specific neoplasia may modulate the same target gene, leading to strong alteration of the molecular pathways downstream the target gene, thus influencing the tumor progression.

RAS oncogene overexpression is associated to miRNA deregulation without oncogene point mutation. Pathway alteration can be induced by downregulation of let-7 miRNA family component.

Regarding the biological processes controlled by miRNAs, they play a direct role on cellular growth control. As previously mentioned, miR-221 is up-regulated in many neoplasms and influence the expression of the cyclin-dependent kinase inhibitor CDKN1B/p27, which controls the cell cycle progression [57]. The activation of pathways involving PI3K/AKT leads to phosphorylation of proteins that favor cell survival, under the control of tumor suppressor lipid phosphatase PTEN, which is a direct miR-21 target; in vitro studies showed the association between mir-21 inhibition and PTEN overexpression, with subsequent decreased neoplastic cell proliferation and invasion [57]. Moreover, MiR-21 inhibition induces changes in metalloproteinases 2 and 9 expression: both these molecules are downstream PTEN mediators with a known role in cellular migration and invasion [57]. These data suggest that the aberrant miR-21 expression may favor HCC growth and diffusion through the modulation of the expression of PTEN and of PTEN-dependent pathways involved in neoplastic cells phenotype and behavior modulation. Again, cyclin G1 upregulation following miR-122 down-regulation may induce p53 down-regulation, so favoring tumorigenesis [57].

Regarding the role of miRNA as biomarkers, there is evidence that, in HCC, miRNAs could have a prognostic as well as a diagnostic role.

As already underlined, some known molecular factors characterizing HCC may play a role as prognostic factors (for example c-MET or p27); similarly, there is evidence that some deregulated miR [58] may allow, in association with clinical parameters and histological patterns, classifying HCC, thus identifying new criteria for prognostic stratification [57]. This is also possible due to distinct miRNA profiles of different hepatocellular carcinoma subtypes (at variance with similar signature between tumors in different organs)Such a differentiation takes place on the basis of supposed mechanism of origin (cancer stem cells or mature hepatocytelike): the different groups show distinctbiological behavior. Furthermore,HBV or HCV etiology of basic disease and primary or metastatic nature of the neoplastic lesion are associated with different miRNA signatures in HCC [60].

Regarding the potential role of miRNAs as diagnostic biomarkers, at least 20 miRNAs show different changes between lesions with different origin; cancer stem cells origin is associated with self-renewal capability, differentiation and aggressive tumorigenesis in vivo: miR-181 family members, over-expressed in HCC, favor the origin of neoplasia from progenitor cells through an influence on CDX2 and GATA6, differentiation related genes, and on NLK a WNT/ Beta catenin pathway inhibitor. 
MiR-21, miR-10b, miR-222 and miR-224 are highly represented in HCC, while non-malignant hepatic lesions show decreased miR-202 and miR-203 expression. Alcohol consumption related hepatocellular carcinoma shows low miR-126 levels, while high levels of miR-96 are present in neoplastic lesions HBV associated: such alterations have not been shown in nonneoplastic parenchyma [58]. Furthermore, hepatocellular carcinoma cases with high risk of metastatic diffusion and cases without metastasis risk present distinct miRNA groups' expression.

Low miR-375 expression have been shown either in hepatocellular adenoma or in carcinoma with Beta catenin mutation: the significant inverse correlation between miR-375 level and Beta catenin targeted gene expression suggests a direct relationship between Beta catenin activation and miR-375 repression [58]. Recently a role of survival predictor and IFN adjuvant therapy response in hepatocellular carcinoma for miR-26 has been identified [77].

Finally, as previously mentioned, genetic mutations and transcriptional, epigenetic changes may induce miRNA alterations. In the case of HCC it has been shown the role of endoribonuclease III DICER for miRNA maturation: its role in cleavage and maturation of miRNA renders impossible for the cell a full expression of miRNA if endoribonuclease is disrupted. DICER deficient hepatocytes loose the expression of all miRNA, with expression of liver specific fetal genes and deregulation of much genes related to neoplastic development [60].

In conclusion, up to date experimental evidences show that hepatocellular carcinoma subtypes are characterized by distinct miRNA expression profiles, related to grade of aggressiveness, risk factors and genetic changes. MiRNAs may therefore represent not only useful diagnostic and prognostic markers, but important target molecules for potential therapeutic treatment.

\section{Tumor associated fibroblast in the development of HCC}

If molecular changes take place in liver parenchymal cells undergoing to inflammatory and fibrotic parenchymal damage, leading to the phenomena that characterize tumoral initiation and progression, then, it is also an important matter the evaluation of the role of inflammatory conditions and fibrosis in stromal changes, and, particularly, in tumor associated fibroblasts development.

Among the constituents of the tumor microenvironment, tumor associated fibroblasts (TAF) play a key role in tumor progression, angiogenesis, growth and metastasis: they are characterized by the expression of specific markers $[45,78-82]$ and seems to assume a role in clinical tumor prognosis.

Recently $[83,84]$ it has been optimized a method to isolate, from several human adult tissues, a population of primitive cells, named multipotent adult stem cells (MASCs) with mesenchymalimmunophenotype, clonogenicity and multiple in vitro differentiation capacity [83, 84]. MASCs isolated both from neoplastic and from cirrhotic human liver tissue allow to test the 
hypothesis that liver resident MASCs could generate TAFs in pathological conditions: a population of MASCs with TAFs characteristics both in human hepatocellular carcinoma and cirrhotic liver has been demonstrated, so suggesting a possible role in the development of neoplasia in the adequate environment [2]. On the other hand, TAFs from non-neoplastic and non-cirrhotic livers do not show aberrant growth properties: so, TAFs can originate from resident primitive cells [2].

The in vivo counterpart of MASCs is still undefined, but the interesting data concern their sharing of some feature with activated hepatic stellate cells [85-87]. This aspect is of particular interest, given the hypothesis that stellate cells could be progenitor cells: in fact, the expression of OCT-4 and of markers of all the three germ layers and their ability to give rise in vitro to endothelial cells and hepatocytes offer essential elements [2].

A fate-mapping study showed that stellate cells could become oval cells when activated in liver injury, and that these cells participate in ductular proliferation [88]. This offers a substantial contribution to the discussed sharing of pathways and cells between developmental, regenerative and neoplastic processes in the liver.

All these evidences point to the presence of mesenchymal, widely multipotent cells in adult tissues, which can take partto regenerative as well as inflammatory and neoplastic processes [89].

In the paper by Cesselli et al 2011 [2] it is well documented that MASCs isolated from hepatocellular carcinoma have the main characteristic of TAFs, at variance with cells from nonneoplastic and non-cirrhotic livers. TAFs act on the tumor growth in different ways, which are able to modify the microenvironment in the sense of a more suitable situation for the increase of tumor growth $[48,78,90]$. TAFs are contractile cells, with a strict spatial relation with blood vessels; they produce growth factors (HGF, TGF beta, EGF, bFGF, IGF), cytokines, chemokines, enzymes; they can degrade the extracellular matrix and can behave as immunomodulating cells $[48,78,90]$. Finally, increased metastatic potential and poor prognosis are associated with the presence of TAFs [48].

Tumor cell lines medium can induce in L-MASCs aberrant growth properties and the ability to produce specific TAF markers [47, 48, 78]. The origin of TAFs seems to be connected with four possible sources: epithelial-mesenchymal transition of the neoplastic cells (associated with genetic changes within the TAFs); recruitment and activation of resident fibroblasts; recruitment of circulating/bone marrow derived mesenchymal stem cells; recruitment of mesenchymal stem cells [48, 78, 82]. In the paper from Cesselli, Beltrami et al. it has been shown the origin of TAFs from a population of resident primitive cells with mesenchymal features [2].The Authors also underline that blocking the activation of TAFs and their continuous communication with the cancer cells could, in conjunction with chemotherapy regimens, limit tumor progression and metastasis [45, 82, 91]: the much lower instability of TAFs versus malignant cells seems to make them less likely to undergo the onset of resistance to chemotherapy drugs [81, 92]. 


\section{Conclusions}

Liver parenchyma, exposed to long standing viral or toxic damage, undergoes a diffuse, fibrosing rearrangement, developing cirrhosis. In this context, genetic changes in the cellular component, the activation of different pathways, the production of cytokines and growth factors, phenotypic changes of epithelial or mesenchymal cells produce complex phenomena, involving regenerative, preneoplastic and neoplastic changes.The role of liver cells and/or progenitor cells in neoplastic growth is heavily influenced by mesenchymal component, in particular by TAFs. Cirrhotic livers, when not yet neoplastic, already possess a population of multipotent adult stem cells with TAFs properties.

The possible origin of TAFs from a population of primitive mesenchymal stem cells gives to the tumor supporting stroma a more complex and important role. In fact, from " rare resident stem cells, such as multipotent adult stem cells (MASCs) " the entire formation and instruction of the elements of supportive microenvironment could take place [2]. MASCs differentiation potential " into many stromal cell types and their molecular signature consisting of overexpression " of a gene panel involved in extracellular matrix remodeling, immunomodulation, and cytokines and growth factors production [83] offer a key to understand the relationship between liver parenchyma, neoplastic growth and stromal component.

"Multipotent adult stem cells isolated from healthy livers can acquire a TAF phenotype when grown in conditional medium from tumor cell lines, suggesting that multipotent cells residing in the liver may represent a population " able to stroma formation and contributing to tumor progression, in adequate conditions [2]. The effort to identify novel therapies aimed at interfering with the interaction between stroma and cancer cells represent a crucial perspective.

The discovery, in 1993, by Ambros and Ruvkun of this new mechanism of gene regulation, with large mRNA under the control of a small RNA opened a new way to develop the understanding of the regulation of critical cellular process (cell division, metabolism, development and death) by micro RNA. MiRNA can target more than 500 mRNA and a single mRNA can be targeted by multiple miRNA. The specificity of targets depend on the degree of base complementarities to the target mRNA: when the miRNA have perfect base complementarities, than messenger RNA undergo degradation; if there is not perfect complementarity, than posttranslational inhibition occur. Through this mechanism, miRNA can exert an essential control on a lot of processes, in particular in neoplasia harboring and diffusion, opening a crucial field in prognostic prediction and in possible intervention to break the carcinogenetic process and to obtain the disease control through target therapy.

The concepts of multidirectional differentiation of mesenchymal stem cells, of tumor associated fibroblasts, of cancerization fields, epithelial-mesenchymal transition and mesenchymalepithelial transition, miRNA deregulation, the progress in the identification of reproducible classification criteria on the basis of hepatocellular carcinomamolecular changes offer new insights concerning liver neoplastic growths, with key points concerning the association between developmental, regenerative and neoplastic growth mechanisms and the relationship between parenchymal hepatocytes and stromal component in preneoplastic and neoplastic liver diseases as the main ways for a better understanding of neoplastic liver biology. 


\section{Author details}

C. Avellini ${ }^{* *}$, D. Cesselli², A.P. Beltrami², M. Orsaria ${ }^{1}$, S. Marzinotto ${ }^{1}$, F. Morassi ${ }^{1}$ and S. Uzzau ${ }^{3}$

*Address all correspondence to: avellini.claudio@aoud.sanita.fvg.it

1 Department of Pathology, University Hospital "S. Maria dellaMisericordia" of Udine, Udine, Italy

2 Department of Medical and Biological Sciences, University of Udine, Udine, Italy

3 Department of Surgery, University Hospital “S. Maria dellaMisericordia” of Udine, Udine, Italy

\section{References}

[1] Thorgeirsson SS, Grisham JW. Molecular pathogenesis of human hepatocellular carcinoma. Nat Genet. 2002 Aug;31(4):339-46.

[2] Cesselli D, Beltrami AP, Poz A, Marzinotto S, Comisso E, Bergamin N, et al. Role of tumor associated fibroblasts in human liver regeneration, cirrhosis, and cancer. Int J Hepatol. 2011;2011:120925.

[3] Mcsween. Pathology of the liver. Livingstone C, editor2002.

[4] Patsenker E, Stickel F. Role of integrins in fibrosing liver diseases. Am J PhysiolGastrointest Liver Physiol. 2011 Sep;301(3):G425-34.

[5] Alison MR, Islam S, Lim S. Stem cells in liver regeneration, fibrosis and cancer: the good, the bad and the ugly. J Pathol. 2009 Jan;217(2):282-98.

[6] Semela D, Dufour JF. Angiogenesis and hepatocellular carcinoma. J Hepatol. 2004 Nov;41(5):864-80.

[7] Sanz-Cameno P, Trapero-Marugan M, Chaparro M, Jones EA, Moreno-Otero R. Angiogenesis: from chronic liver inflammation to hepatocellular carcinoma. J Oncol. 2010;2010:272170.

[8] Kung JW, Currie IS, Forbes SJ, Ross JA. Liver development, regeneration, and carcinogenesis. J Biomed Biotechnol. 2010;2010:984248.

[9] Riehle KJ, Dan YY, Campbell JS, Fausto N. New concepts in liver regeneration. J GastroenterolHepatol. 2011 Jan;26Suppl 1:203-12. 
[10] Jung J, Zheng M, Goldfarb M, Zaret KS. Initiation of mammalian liver development from endoderm by fibroblast growth factors. Science. 1999 Jun 18;284(5422): 1998-2003.

[11] Rossi JM, Dunn NR, Hogan BL, Zaret KS. Distinct mesodermal signals, including BMPs from the septum transversum mesenchyme, are required in combination for hepatogenesis from the endoderm. Genes Dev. 2001 Aug 1;15(15):1998-2009.

[12] Deutsch G, Jung J, Zheng M, Lora J, Zaret KS. A bipotential precursor population for pancreas and liver within the embryonic endoderm. Development. 2001 Mar;128(6): 871-81.

[13] Shin D, Shin CH, Tucker J, Ober EA, Rentzsch F, Poss KD, et al. Bmp and Fgf signaling are essential for liver specification in zebrafish. Development. 2007 Jun;134(11): 2041-50.

[14] Zhang W, Yatskievych TA, Baker RK, Antin PB. Regulation of Hex gene expression and initial stages of avian hepatogenesis by Bmp and Fgf signaling. Dev Biol. 2004 Apr 15;268(2):312-26.

[15] McLin VA, Rankin SA, Zorn AM. Repression of Wnt/beta-catenin signaling in the anterior endoderm is essential for liver and pancreas development. Development. 2007 Jun;134(12):2207-17.

[16] Monga SP, Monga HK, Tan X, Mule K, Pediaditakis P, Michalopoulos GK. Beta-catenin antisense studies in embryonic liver cultures: role in proliferation, apoptosis, and lineage specification. Gastroenterology. 2003 Jan;124(1):202-16.

[17] Ober EA, Verkade H, Field HA, Stainier DY. Mesodermal Wnt2b signalling positively regulates liver specification. Nature. 2006 Aug 10;442(7103):688-91.

[18] Schmidt C, Bladt F, Goedecke S, Brinkmann V, Zschiesche W, Sharpe M, et al. Scatter factor/hepatocyte growth factor is essential for liver development. Nature. $1995 \mathrm{Feb}$ 23;373(6516):699-702.

[19] Tanimizu N, Miyajima A. Molecular mechanism of liver development and regeneration. Int Rev Cytol. 2007;259:1-48.

[20] Weinstein M, Monga SP, Liu Y, Brodie SG, Tang Y, Li C, et al. Smad proteins and hepatocyte growth factor control parallel regulatory pathways that converge on beta1-integrin to promote normal liver development. Mol Cell Biol. 2001 Aug;21(15): 5122-31.

[21] Zhao R, Duncan SA. Embryonic development of the liver. Hepatology. 2005 May; 41(5):956-67.

[22] Kuwahara R, Kofman AV, Landis CS, Swenson ES, Barendswaard E, Theise ND. The hepatic stem cell niche: identification by label-retaining cell assay. Hepatology. 2008 Jun;47(6):1994-2002. 
[23] Fausto N, Campbell JS, Riehle KJ. Liver regeneration. Hepatology. 2006 Feb;43(2 Suppl 1):S45-53.

[24] Diehl AM. Recent events in alcoholic liver disease V. effects of ethanol on liver regeneration. Am J PhysiolGastrointest Liver Physiol. 2005 Jan;288(1):G1-6.

[25] Roskams T. Liver stem cells and their implication in hepatocellular and cholangiocarcinoma. Oncogene. 2006 Jun 26;25(27):3818-22.

[26] Roskams T. Different types of liver progenitor cells and their niches. J Hepatol. 2006 Jul;45(1):1-4.

[27] Riehle KJ, Dan YY, Campbell JS, Fausto N. New concepts in liver regeneration. J GastroenterolHepatol.Jan;26Suppl 1:203-12.

[28] Nagy P, Bisgaard HC, Santoni-Rugiu E, Thorgeirsson SS. In vivo infusion of growth factors enhances the mitogenic response of rat hepatic ductal (oval) cells after administration of 2-acetylaminofluorene. Hepatology. 1996 Jan;23(1):71-9.

[29] Hasuike S, Ido A, Uto H, Moriuchi A, Tahara Y, Numata M, et al. Hepatocyte growth factor accelerates the proliferation of hepatic oval cells and possibly promotes the differentiation in a 2-acetylaminofluorene/partial hepatectomy model in rats. J GastroenterolHepatol. 2005 Nov;20(11):1753-61.

[30] Isfort RJ, Cody DB, Stuard SB, Randall CJ, Miller C, Ridder GM, et al. The combination of epidermal growth factor and transforming growth factor-beta induces novel phenotypic changes in mouse liver stem cell lines. J Cell Sci. 1997 Dec;110 ( Pt 24): 3117-29.

[31] Nguyen LN, Furuya MH, Wolfraim LA, Nguyen AP, Holdren MS, Campbell JS, et al. Transforming growth factor-beta differentially regulates oval cell and hepatocyte proliferation. Hepatology. 2007 Jan;45(1):31-41.

[32] Knight B, Tirnitz-Parker JE, Olynyk JK. C-kit inhibition by imatinibmesylate attenuates progenitor cell expansion and inhibits liver tumor formation in mice. Gastroenterology. 2008 Sep;135(3):969-79, 79 e1.

[33] Sell S, Leffert HL. An evaluation of cellular lineages in the pathogenesis of experimental hepatocellular carcinoma. Hepatology. 1982 Jan-Feb;2(1):77-86.

[34] Sell S, Leffert HL. Liver cancer stem cells. J ClinOncol. 2008 Jun 10;26(17):2800-5.

[35] Lee JS, Heo J, Libbrecht L, Chu IS, Kaposi-Novak P, Calvisi DF, et al. A novel prognostic subtype of human hepatocellular carcinoma derived from hepatic progenitor cells. Nat Med. 2006 Apr;12(4):410-6.

[36] Suzuki A, Sekiya S, Onishi M, Oshima N, Kiyonari H, Nakauchi H, et al. Flow cytometric isolation and clonal identification of self-renewing bipotent hepatic progenitor cells in adult mouse liver. Hepatology. 2008 Dec;48(6):1964-78. 
[37] Chiba T, Kita K, Zheng YW, Yokosuka O, Saisho H, Iwama A, et al. Side population purified from hepatocellular carcinoma cells harbors cancer stem cell-like properties. Hepatology. 2006 Jul;44(1):240-51.

[38] Yamashita T, Ji J, Budhu A, Forgues M, Yang W, Wang HY, et al. EpCAM-positive hepatocellular carcinoma cells are tumor-initiating cells with stem/progenitor cell features. Gastroenterology. 2009 Mar;136(3):1012-24.

[39] Yamashita T, Forgues M, Wang W, Kim JW, Ye Q, Jia H, et al. EpCAM and alphafetoprotein expression defines novel prognostic subtypes of hepatocellular carcinoma. Cancer Res. 2008 Mar 1;68(5):1451-61.

[40] Durnez A, Verslype C, Nevens F, Fevery J, Aerts R, Pirenne J, et al. The clinicopathological and prognostic relevance of cytokeratin 7 and 19 expression in hepatocellular carcinoma. A possible progenitor cell origin. Histopathology. 2006 Aug;49(2):138-51.

[41] Duncan AW, Dorrell C, Grompe M. Stem cells and liver regeneration. Gastroenterology. 2009 Aug;137(2):466-81.

[42] Herrera MB, Bruno S, Buttiglieri S, Tetta C, Gatti S, Deregibus MC, et al. Isolation and characterization of a stem cell population from adult human liver. Stem Cells. 2006 Dec;24(12):2840-50.

[43] Dvorak HF. Tumors: wounds that do not heal. Similarities between tumor stroma generation and wound healing. N Engl J Med. 1986 Dec 25;315(26):1650-9.

[44] Hanahan D, Weinberg RA. The hallmarks of cancer. Cell. 2000 Jan 7;100(1):57-70.

[45] Kalluri R, Zeisberg M. Fibroblasts in cancer. Nat Rev Cancer. 2006 May;6(5):392-401.

[46] Pietras K, Ostman A. Hallmarks of cancer: interactions with the tumor stroma. Exp Cell Res. 2010 May 1;316(8):1324-31.

[47] Rasanen K, Vaheri A. Activation of fibroblasts in cancer stroma. Exp Cell Res. 2010 Oct 15;316(17):2713-22.

[48] Shimoda M, Mellody KT, Orimo A. Carcinoma-associated fibroblasts are a rate-limiting determinant for tumour progression. Semin Cell Dev Biol. 2010 Feb;21(1):19-25.

[49] Anderson AR, Weaver AM, Cummings PT, Quaranta V. Tumor morphology and phenotypic evolution driven by selective pressure from the microenvironment. Cell. 2006 Dec 1;127(5):905-15.

[50] Mishra P, Banerjee D, Ben-Baruch A. Chemokines at the crossroads of tumor-fibroblast interactions that promote malignancy. J Leukoc Biol. 2010 Jan;89(1):31-9.

[51] Polyak K, Haviv I, Campbell IG. Co-evolution of tumor cells and their microenvironment. Trends Genet. 2009 Jan;25(1):30-8. 
[52] Chang HY, Sneddon JB, Alizadeh AA, Sood R, West RB, Montgomery K, et al. Gene expression signature of fibroblast serum response predicts human cancer progression: similarities between tumors and wounds. PLoS Biol. 2004 Feb;2(2):E7.

[53] Thiery JP, Sleeman JP. Complex networks orchestrate epithelial-mesenchymal transitions. Nat Rev Mol Cell Biol. 2006 Feb;7(2):131-42.

[54] Farazi PA, DePinho RA. Hepatocellular carcinoma pathogenesis: from genes to environment. Nat Rev Cancer. 2006 Sep;6(9):674-87.

[55] Bruix J, Boix L, Sala M, Llovet JM. Focus on hepatocellular carcinoma. Cancer Cell. 2004 Mar;5(3):215-9.

[56] Hoshida Y, Toffanin S, Lachenmayer A, Villanueva A, Minguez B, Llovet JM. Molecular classification and novel targets in hepatocellular carcinoma: recent advancements. Semin Liver Dis. 2010 Feb;30(1):35-51.

[57] Gramantieri L, Fornari F, Callegari E, Sabbioni S, Lanza G, Croce CM, et al. MicroRNA involvement in hepatocellular carcinoma. J Cell Mol Med. 2008 Dec;12(6A): 2189-204.

[58] Ladeiro Y, Couchy G, Balabaud C, Bioulac-Sage P, Pelletier L, Rebouissou S, et al. MicroRNA profiling in hepatocellular tumors is associated with clinical features and oncogene/tumor suppressor gene mutations. Hepatology. 2008 Jun;47(6):1955-63.

[59] McManus MT. MicroRNAs and cancer. Semin Cancer Biol. 2003 Aug;13(4):253-8.

[60] Ji J, Wang XW. New kids on the block: diagnostic and prognostic microRNAs in hepatocellular carcinoma. Cancer BiolTher. 2009 Sep;8(18):1686-93.

[61] Calin GA, Croce CM. MicroRNA signatures in human cancers. Nat Rev Cancer. 2006;6:857-66.

[62] Weber B, Stresemann C, Brueckner B, Lyko F. Methylation of human microRNA genes in normal and neoplastic cells. Cell Cycle. 2007 May 2;6(9):1001-5.

[63] Fazi F, Racanicchi S, Zardo G, Starnes LM, Mancini M, Travaglini L, et al. Epigenetic silencing of the myelopoiesis regulator microRNA-223 by the AML1/ETO oncoprotein. Cancer Cell. 2007 Nov;12(5):457-66.

[64] Volinia S, Calin GA, Liu CG, Ambs S, Cimmino A, Petrocca F, et al. A microRNA expression signature of human solid tumors defines cancer gene targets. ProcNatlAcadSci U S A. 2006 Feb 14;103(7):2257-61.

[65] Ma L, Teruya-Feldstein J, Weinberg RA. Tumour invasion and metastasis initiated by microRNA-10b in breast cancer. Nature. 2007 Oct 11;449(7163):682-8.

[66] Li N, Fu H, Tie Y, Hu Z, Kong W, Wu Y, et al. miR-34a inhibits migration and invasion by down-regulation of c-Met expression in human hepatocellular carcinoma cells. Cancer Lett. 2009 Mar 8;275(1):44-53. 
[67] Esau C, Davis S, Murray SF, Yu XX, Pandey SK, Pear M, et al. miR-122 regulation of lipid metabolism revealed by in vivo antisense targeting. Cell Metab. 2006 Feb;3(2): 87-98.

[68] Krutzfeldt J, Rajewsky N, Braich R, Rajeev KG, Tuschl T, Manoharan M, et al. Silencing of microRNAs in vivo with 'antagomirs'. Nature. 2005 Dec 1;438(7068):685-9.

[69] Jopling CL, Yi M, Lancaster AM, Lemon SM, Sarnow P. Modulation of hepatitis C virus RNA abundance by a liver-specific MicroRNA. Science. 2005 Sep 2;309(5740): 1577-81.

[70] Murakami Y, Yasuda T, Saigo K, Urashima T, Toyoda H, Okanoue T, et al. Comprehensive analysis of microRNA expression patterns in hepatocellular carcinoma and non-tumorous tissues. Oncogene. 2006 Apr 20;25(17):2537-45.

[71] Budhu A, Jia HL, Forgues M, Liu CG, Goldstein D, Lam A, et al. Identification of metastasis-related microRNAs in hepatocellular carcinoma. Hepatology. 2008 Mar; 47(3):897-907.

[72] Pei $Y$, Zhang T, Renault V, Zhang X. An overview of hepatocellular carcinoma study by omics-based methods. ActaBiochimBiophys Sin (Shanghai). 2009 Jan;41(1):1-15.

[73] Meng F, Henson R, Wehbe-Janek H, Ghoshal K, Jacob ST, Patel T. MicroRNA-21 regulates expression of the PTEN tumor suppressor gene in human hepatocellular cancer. Gastroenterology. 2007 Aug;133(2):647-58.

[74] Wang Y, Lee AT, Ma JZ, Wang J, Ren J, Yang Y, et al. Profiling microRNA expression in hepatocellular carcinoma reveals microRNA-224 up-regulation and apoptosis inhibitor-5 as a microRNA-224-specific target. J Biol Chem. 2008 May 9;283(19): 13205-15.

[75] Ura S, Honda M, Yamashita T, Ueda T, Takatori H, Nishino R, et al. Differential microRNA expression between hepatitis $\mathrm{B}$ and hepatitis $\mathrm{C}$ leading disease progression to hepatocellular carcinoma. Hepatology. 2009 Apr;49(4):1098-112.

[76] Kim S, Lee UJ, Kim MN, Lee EJ, Kim JY, Lee MY, et al. MicroRNA miR-199a* regulates the MET proto-oncogene and the downstream extracellular signal-regulated kinase 2 (ERK2). J Biol Chem. 2008 Jun 27;283(26):18158-66.

[77] Roessler S, Jia HL, Budhu A, Forgues M, Ye QH, Lee JS, et al. A unique metastasis gene signature enables prediction of tumor relapse in early-stage hepatocellular carcinoma patients. Cancer Res. 2010 Dec 15;70(24):10202-12.

[78] Spaeth EL, Dembinski JL, Sasser AK, Watson K, Klopp A, Hall B, et al. Mesenchymal stem cell transition to tumor-associated fibroblasts contributes to fibrovascular network expansion and tumor progression. PLoS One. 2009;4(4):e4992.

[79] Mishra PJ, Glod JW, Banerjee D. Mesenchymal stem cells: flip side of the coin. Cancer Res. 2009 Feb 15;69(4):1255-8. 
[80] Mishra PJ, Humeniuk R, Medina DJ, Alexe G, Mesirov JP, Ganesan S, et al. Carcinoma-associated fibroblast-like differentiation of human mesenchymal stem cells. Cancer Res. 2008 Jun 1;68(11):4331-9.

[81] Mueller MM, Fusenig NE. Friends or foes -bipolar effects of the tumourstroma in cancer. Nat Rev Cancer. 2004 Nov;4(11):839-49.

[82] Wels J, Kaplan RN, Rafii S, Lyden D. Migratory neighbors and distant invaders: tumor-associated niche cells. Genes Dev. 2008 Mar 1;22(5):559-74.

[83] Beltrami AP, Cesselli D, Bergamin N, Marcon P, Rigo S, Puppato E, et al. Multipotent cells can be generated in vitro from several adult human organs (heart, liver, and bone marrow). Blood. 2007 Nov 1;110(9):3438-46.

[84] Cesselli D, Beltrami AP, Rigo S, Bergamin N, D'Aurizio F, Verardo R, et al. Multipotent progenitor cells are present in human peripheral blood. Circ Res. 2009 May 22;104(10):1225-34.

[85] Kordes C, Sawitza I, Haussinger D. Canon-icalWnt signaling maintains the quiescent stage of hepatic stellate cells. BiochemBiophys Res Commun. 2008 Feb 29;367(1): $116-23$.

[86] Kordes C, Sawitza I, Muller-Marbach A, Ale-Agha N, Keitel V, Klonowski-Stumpe $\mathrm{H}$, et al. CD133+ hepatic stellate cells are progenitor cells. BiochemBiophys Res Commun. 2007 Jan 12;352(2):410-7.

[87] Kordes C, Sawitza I, Haussinger D. Hepatic and pancreatic stellate cells in focus. Biol Chem. 2009 Oct;390(10):1003-12.

[88] Yang L, Jung Y, Omenetti A, Witek RP, Choi S, Vandongen HM, et al. Fate-mapping evidence that hepatic stellate cells are epithelial progenitors in adult mouse livers. Stem Cells. 2008 Aug;26(8):2104-13.

[89] Slack JM. Origin of stem cells in organogenesis. Science. 2008 Dec 5;322(5907): 1498-501.

[90] Franco OE, Shaw AK, Strand DW, Hayward SW. Cancer associated fibroblasts in cancer pathogenesis. Semin Cell Dev Biol. 2010 Feb;21(1):33-9.

[91] LeBeau AM, Brennen WN, Aggarwal S, Denmeade SR. Targeting the cancer stroma with a fibroblast activation protein-activated promelittinprotoxin. Mol Cancer Ther. 2009 May;8(5):1378-86.

[92] Orimo A, Weinberg RA. Stromal fibroblasts in cancer: a novel tumor-promoting cell type. Cell Cycle. 2006 Aug;5(15):1597-601. 
